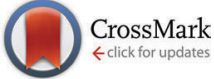

Cite this: Chem. Commun., 2015 51, 4619

Received 30th December 2014, Accepted 10th February 2015

DOI: $10.1039 / c 4 c c 10401 c$

www.rsc.org/chemcomm

\section{An antibody-graphene oxide nanoribbon conjugate as a surface enhanced laser desorption/ionization probe with high sensitivity and selectivity $\dagger$}

\author{
Jing Wang, ${ }^{\text {ab }}$ Mengting Cheng, ${ }^{a}$ Zhen Zhang, ${ }^{c}$ Liangqia Guo, ${ }^{b}$ Qian Liu*a and \\ Guibin Jiang ${ }^{a}$
}

\begin{abstract}
Graphene oxide nanoribbons (GONRs) were covalently functionalized with an antibody using polyethylene glycol (PEG) as a linker to produce a novel probe for surface enhanced laser desorption/ionization mass spectrometry (SELDI MS). This probe provides a highly sensitive and selective platform for enrichment and MS detection of small molecules in complex media.
\end{abstract}

Detection of biologically important small molecules in complex media by mass spectrometry (MS) is of extreme importance. MS offers high sensitivity, reliability, and outstanding qualitative ability. However, its application is seriously hindered by the ionization suppression effect and matrix interferences. As a result, complicated sample preparation procedures are often required prior to MS analysis. Surface-enhanced laser desorption/ionization (SELDI), a derivant mode of matrix-assisted laser desorption/ionization (MALDI), provides a valuable technique for MS analysis. ${ }^{1}$ SELDI uses a probe for extraction, enrichment, desorption, and ionization of the target analytes. The probe serves as both an extractor and a MALDI matrix, thus avoiding the use of an organic matrix and cutting the time and cost for analysis.

Graphene (G), a two-dimensional and one-atom-thick carbon structure, ${ }^{2}$ has emerged as a good probe for SELDI MS. ${ }^{3}$ G has a high loading capacity for analyte extraction and enrichment due to its ultra-large surface area. ${ }^{4}$ Furthermore, the universal and frequency-independent optical absorption of $\mathrm{G}$ makes it a highly efficient matrix for MALDI. ${ }^{5}$ However, a big problem in the application of $\mathrm{G}$ is the specificity. $\mathrm{G}$ has strong adsorption capabilities for a broad range of compounds. In real complex media, competitive adsorption of non-target compounds on $\mathrm{G}$ may greatly decrease the response of the target analytes and compromise the exceptional

\footnotetext{
${ }^{a}$ State Key Laboratory of Environmental Chemistry and Ecotoxicology, Research Center for Eco-Environmental Sciences, Chinese Academy of Sciences, Beijing 100085, China. E-mail: qianliu@rcees.ac.cn

${ }^{b}$ Department of Chemistry, Fuzhou University, Fuzhou 350002, China

${ }^{c}$ School of the Environment and Safety Engineering, Jiangsu University, Zhenjiang 212013, China

$\dagger$ Electronic supplementary information (ESI) available. See DOI: 10.1039/ c4cc10401c
}

properties of G. This problem exists not only when $\mathrm{G}$ is used as a SELDI probe but also in other application aspects. Gulbakan et al. used nucleic acid aptamers to modify graphene oxide (GO) for analyte enrichment and MS readout. ${ }^{6}$ However, aptamers are available only for a limited number of analytes and their binding ability in complex media is still a concern.

Here we report the synthesis of antibody-functionalized graphene oxide nanoribbons (GONR-PEG-Ab) using polyethylene glycol (PEG) as a linker and their application as SELDI probes. As an antibody can recognize and specifically bind with the antigen, modification with an antibody can greatly improve the specificity of the probe. Chloramphenicol (CAP), a widely used antibiotic, was used as a model analyte. Notably, we used graphene oxide nanoribbons (GONRs), a novel variety of $\mathrm{G},{ }^{7}$ as scaffolds for the construction of G-antibody conjugates rather than normal G sheets, due to the following considerations: (1) GONRs are regarded as low-defect $\mathrm{G}$ materials. ${ }^{8}$ GONRs are synthesized by longitudinal unzipping of multi-walled carbon nanotubes (MWCNTs) through chemical oxidation. ${ }^{7 b}$ The oxidation sites are supposed to mainly locate at the edges of GONRs without sacrificing the integrity of the $\pi$-conjugation network in basal planes. ${ }^{8 a}$ This structure can retain the high energy absorption and transfer capabilities of G for SELDI application. (2) The oxygen-containing groups in GONRs can improve the water dispersibility and provide ready sites for further functionalization. (3) GONRs are more monodisperse in geometry than normal G sheets, which should be more favorable for homogenous chemical modification and obtaining reproducible analytical results. Therefore, GONRs are expected to achieve better performance than normal $\mathrm{G}$ in SELDI.

The synthetic procedures for GONR-PEG-Ab are depicted in Fig. 1. The anti-CAP monoclonal antibody was prepared and purified in the lab using the hybridoma technique (see the ESI $\uparrow$ for details). ${ }^{9} \mathrm{~A}$ bifunctional PEG $\left(\mathrm{NH}_{2}-\mathrm{PEG}-\mathrm{COOH}\right)$ was used as a linker for GONRs and the antibody. It is well-known that the function of an antibody relies on its specific tertiary or quaternary structure. ${ }^{10}$ To prevent possible loss of activity of the antibody after conjugation to GONRs, PEG was used as a spacer to maintain the steric structure of the antibody. The amino end of $\mathrm{NH}_{2}-\mathrm{PEG}-\mathrm{COOH}$ 


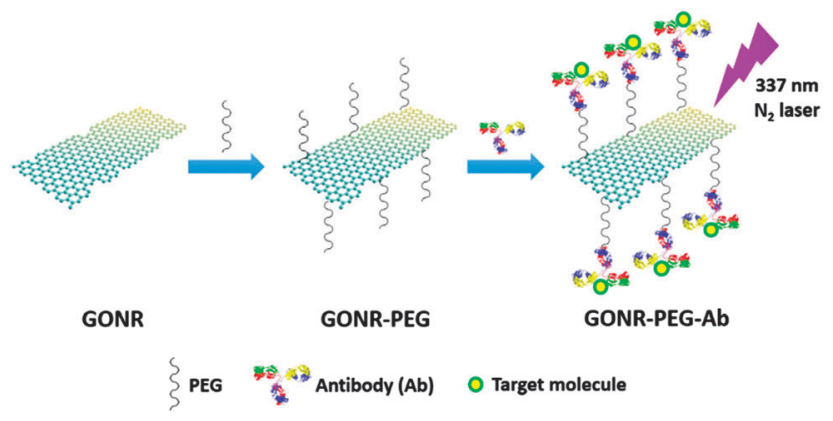

Fig. 1 Scheme showing the synthetic procedures of GONR-PEG-Ab and its use as a probe in SELDI-TOF MS.

was firstly bound to the carboxyl groups of GONRs by using EDC/NHS ( $N$-(3-dimethylaminopropyl)- $N$-ethylcarbodiimide hydrochloride/ $N$-hydroxysuccinimide) as a coupling agent. Then, the antibody was covalently attached to the PEGylated GONRs by coupling the amino groups of the antibody with the carboxyl end of $\mathrm{NH}_{2}-\mathrm{PEG}-\mathrm{COOH}$.

The as-prepared GONR-PEG-Ab was characterized by different techniques. Fig. 2A and B show the tapping-mode AFM images of
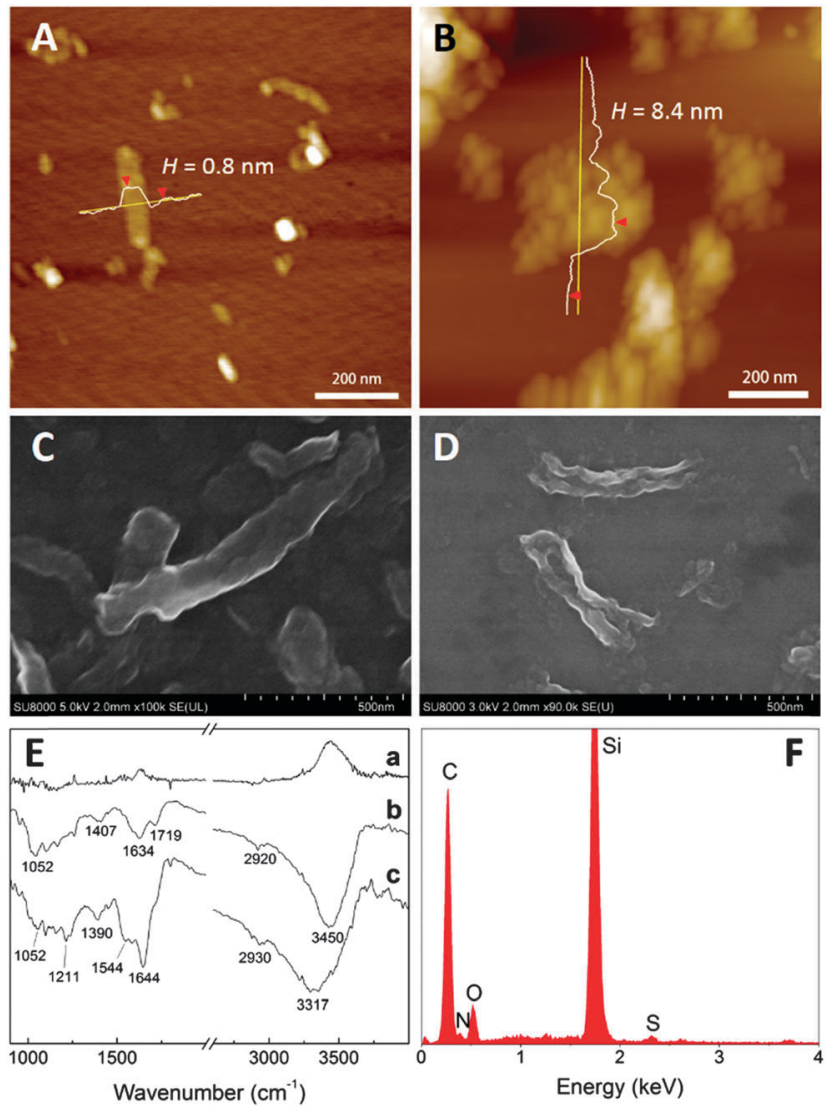

Fig. 2 Characterization of materials. (A, B) AFM images of GONRs (A) and GONR-PEG-Ab (B). Height profiles taken along the yellow lines indicate thickness of 0.8 and $8.4 \mathrm{~nm}$, respectively. (C, D) SEM images of GONRs (C) and GONR-PEG-Ab (D). (E) FT-IR spectra of MWCNTs (a), GONRs (b), and GONRPEG-Ab (c). (F) EDX spectrum of GONR-PEG-Ab. The spectrum was obtained using silicon plate as a substrate, so a strong Si peak was observed.
GONRs and GONR-PEG-Ab, respectively. A typical height profile of GONRs indicates a thickness of $0.8 \mathrm{~nm}$, suggesting the singlelayer nature of the obtained GONRs. After conjugation with the antibody $\left(M_{\mathrm{w}} \sim 150 \mathrm{kDa}\right)$, the thickness increases to $8.4 \mathrm{~nm}$. The SEM images show that the MWCNTs have been completely unravelled by oxidation to produce GONRs (Fig. 2C). The width of the GONRs can exceed $160 \mathrm{~nm}$. After being functionalized with PEG and the antibody, GONR-PEG-Ab can maintain the morphology of nanoribbons (Fig. 2D). No aggregation was observed in either GONRs or GONR-PEG-Ab (Fig. S1, ESI $\dagger$ ). Fig. 2E shows the FT-IR spectra of MWCNTs, GONRs, and GONR-PEG-Ab. MWCNTs show no significant feature peaks (a). After oxidation to GONRs (b), a broad and strong absorption band at $3450 \mathrm{~cm}^{-1}$ is observed due to the $\mathrm{O}-\mathrm{H}$ stretching vibration. The adsorptions at 1719 and $1634 \mathrm{~cm}^{-1}$ are assigned to the $\mathrm{C}=\mathrm{O}$ stretching of carboxyl groups and the $\mathrm{O}-\mathrm{H}$ bending and skeletal ring vibrations, respectively. In the spectrum of GONR-PEG-Ab (c), the strong absorption at $3450 \mathrm{~cm}^{-1}$ shifts to $3317 \mathrm{~cm}^{-1}$ due to the $\mathrm{N}-\mathrm{H}$ stretching vibration, and the peaks at $2930 \mathrm{~cm}^{-1}$ are enhanced due to the asymmetric stretching vibrations of the methylenes in PEG chains. Notably, a strong peak appears at $1644 \mathrm{~cm}^{-1}$ corresponding to the $\mathrm{C}=\mathrm{O}$ stretching of amide groups, which indicates the effectiveness of the coupling reaction. Fig. 2F shows the EDX spectrum of GONR-PEG-Ab. Compared to those of MWCNTs and GONRs (Fig. S2, ESI $\dagger$ ), new peaks of $\mathrm{N}$ and $\mathrm{S}$ are observed. The antibody is the sole source of $\mathrm{S}$, so the presence of the $\mathrm{S}$ peak indicates the introduction of the antibody. The synthetic process was also monitored using TEM, UV-visible absorption spectrometry, XPS, and XRD (see Fig. S3-S6, ESI $\dagger$ ).

Prior to application in SELDI, we first tested the performance of GONRs as a matrix in MALDI MS to justify the use of GONRs. The CAP solution was mixed with the GONR dispersion, and then the mixture was directly used for MALDI-TOF MS measurement without extraction. For comparison, the measurements were also carried out with MWCNTs, G, GO, and a conventional organic matrix CHCA ( $\alpha$-cyano-4-hydroxycinnamic acid) as matrices. We found that dual peaks of $[\mathrm{M}-\mathrm{H}]^{-}$of CAP could be detected at $m / z 320.4$ and 322.4, and GONRs generated the strongest MS signals among the tested materials (Fig. S7, ESI $\dagger$ ). The peak intensity decreased in the following order: GONR $>\mathrm{G}>$ MWCNT $>$ GO $\approx$ CHCA. This proves our hypothesis that GONR is a highly efficient matrix for MALDI. The high performance of GONRs is mainly ascribed to two reasons: (1) the unique lowdefect $\mathrm{G}$ structure in GONRs, as described above, keeping strong optical absorption and energy transfer capability in MALDI; (2) the good dispersibility of GONRs. It has been reported that the dispersed state of the matrix has a significant impact on the MS signals. ${ }^{5 b, 11}$ We investigated the morphology of the matrix on the MALDI target by SEM (Fig. S8, ESI $\dagger$ ), and found that GONRs can form a flat and homogenous film on the MALDI target due to their good water-dispersibility, which is favorable for energy absorption and transfer in LDI. In contrast, the G matrix aggregates or re-stacks to form discrete clusters, thereby reducing its performance and decreasing the coverage of the MALDI target. It should be noted that GONR-PEG-Ab could also form a homogenous film on the MALDI target, indicating that the functionalization 
does not affect the dispersibility of GONRs. Accordingly, the high performance of GONRs in MALDI satisfies the prerequisite for the use of GONR-PEG-Ab as a SELDI probe.

To perform the SELDI MS analysis, $50 \mu \mathrm{L}$ of the probe dispersion $\left(0.5 \mathrm{mg} \mathrm{mL}^{-1}\right)$ was added to $5 \mathrm{~mL}$ of the sample solution to extract and enrich the target analytes. The probe was then isolated from the solution by centrifugal ultrafiltration and used for MS measurement. For comparison, we have also synthesized GONR-Ab (without the PEG linker) and GONR-PEG (without the antibody) and tested them as SELDI probes. The method was first evaluated using pure water samples (Fig. S9, ESI $\dagger$ ). In unspiked water samples, no feature peaks of CAP were detected. When spiked with CAP at $1 \mathrm{ng} \mathrm{mL}{ }^{-1}$, the peaks of $[\mathrm{M}-\mathrm{H}]^{-}$of CAP could be readily detected. Since in pure water there is no matrix interference, all the tested probes (GONR-PEG-Ab, GONR-Ab, GONR-PEG, and GONR) could detect CAP at $1 \mathrm{ng} \mathrm{mL}{ }^{-1}$. Despite that, GONR-PEG-Ab showed stronger MS signals than the other probes, demonstrating that GONR-PEG-Ab can more effectively capture the target analyte and that the performance of GONRs in LDI can be preserved after modification with the antibody.

The advantages of GONR-PEG-Ab can be demonstrated more clearly in complex media. Herein, we chose human serum and river water as sample matrices. Antibiotics have recently been regarded as a class of emerging pollutants due to their abuse and increasing disposal level into the environment. ${ }^{12}$ So development of detection methods for antibiotics in environmental media is very important. We compared the performance of different materials (GONR-PEG-Ab, GONR-Ab, GONR-PEG, and GONR) as SELDI probes in the detection of $1 \mathrm{ng} \mathrm{mL}^{-1}$ CAP in river water and human serum samples. The results are shown in Fig. 3. The intensities and $\mathrm{S} / \mathrm{N}$ (signal-to-noise ratio) of the feature peaks of CAP are given in Table S1, ESI. $\dagger$ As shown in Fig. 3I and J, $1 \mathrm{ng} \mathrm{mL}^{-1}$ CAP cannot be detected by MS without using a probe due to insufficient sensitivity. A large number of noise peaks are observed in the spectra due to complex sample matrices. Using bare GONR as the probe (Fig. 3G and H), no feature peaks are obtained for river water samples; in the serum samples CAP can be detected but the peak intensity is low. This suggests that GONR lacks the specificity to overcome the serious matrix interferences in these complex samples. GONR-PEG produces more noise peaks in river water samples (Fig. 3E) and shows no improvement than GONR in serum samples (Fig. 3F). When using GONR-Ab as a probe (Fig. 3C and D), CAP can be detected in both river water and serum samples, and the noise peaks can be significantly suppressed. However, the peak intensities are still low, indicating that the PEG linker plays an important role in the probe to retain the activity of the antibody. Only when using GONR-PEG-Ab as a probe (Fig. 3A and $\mathrm{B}$ ), the highest peak intensities are obtained. For example, the signal intensity obtained with GONR-PEG-Ab in serum samples is 5.1, 5.1, and 2.8 times higher than that obtained with GONR-Ab, GONR-PEG, and GONR, respectively. These results definitely demonstrate the high efficiency and selectivity of GONR-PEG-Ab probe in complex media.

It should be noted that whether a protein can retain its function after binding to $\mathrm{G}$ is still an active research topic.

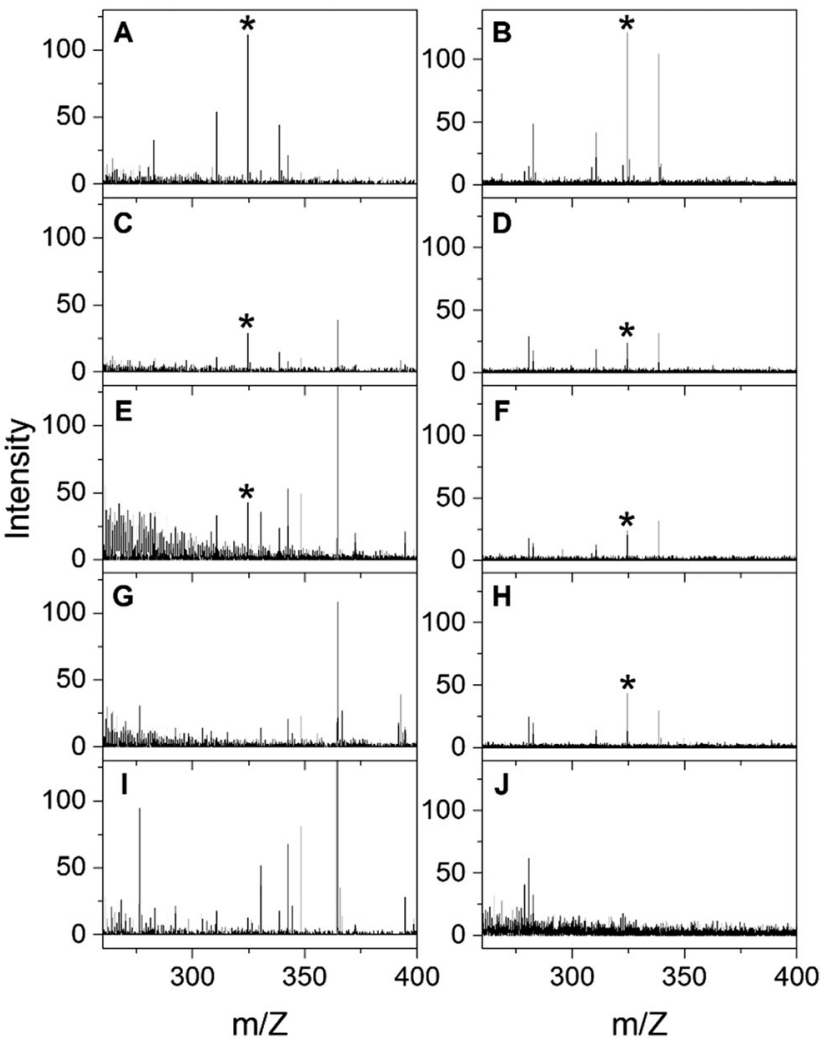

Fig. 3 Detection of CAP in river water (left column) and human serum samples (right column) by SELDI-TOF MS using different materials as probes. (A, B) GONR-PEG-Ab, (C, D) GONR-Ab, (E, F) GONR-PEG, (G, H) GONRs, (I, J) direct analysis without using probe. CAP spike concentration: $1 \mathrm{ng} \mathrm{mL}{ }^{-1}$. The feature peak of CAP was marked with asterisk.

Although some papers have reported that proteins may undergo significant conformational changes and lose some of the functions when adsorbing to $\mathrm{G}$ materials, ${ }^{10,13}$ in applications this point was often not taken into consideration. ${ }^{14}$ Our observation that GONR-Ab produces much lower signals than GONR-PEG-Ab suggests that the antibody indeed loses part of its affinity if it is directly bound to GONRs. Using a spacer (i.e., PEG) can effectively control the antibody adsorption to GONRs and thus recover its activity. This demonstrates the necessity of engineering the interfaces in designing G-protein conjugates.

The high efficiency and selectivity of GONR-PEG-Ab result in high sensitivity. The detection limit (LOD, at S/N 3) of CAP by SELDI MS in river water and serum samples was determined to be $10 \mathrm{pg} \mathrm{mL}^{-1}$. This value is better than that achieved with amplified enzyme-linked immunosorbent assay (ELISA) and gas chromatography-MS, ${ }^{15}$ and comparable or inferior to that obtained with liquid chromatography-ion trap MS and liquid chromatography-electrospray ionization tandem MS. ${ }^{16}$ Meanwhile, the present method is much simpler, quicker, and lower-cost than those methods. The enrichment factor was determined to be $\sim 200$ (see the $\mathrm{ESI} \dagger$ for details), which is higher than that achieved on a commercial solid-phase extraction cartridge. ${ }^{17}$ The dynamic range of detection is $10-1000 \mathrm{pg} \mathrm{mL}^{-1}$. Furthermore, good reproducibility of analysis was obtained. The shot-to-shot RSD $(n=17)$ at different locations on the GONR-PEG-Ab 
matrix was $7.9 \%$, and the sample-to-sample $\operatorname{RSD}(n=5)$ in SELDI analysis was $18.4 \%$. These results indicate that GONRPEG-Ab is a highly sensitive and reliable probe for SELDI.

In summary, we have synthesized antibody-functionalized GONRs and shown their application as SELDI probes in complex media. The probes combine the specificity of an antibody and the exceptional properties of GONRs, thus allowing direct MS detection of small molecules in complex media without the use of any complicated sample preparation process. The analysis is facile, fast, sensitive, and selective. Therefore, the GONR-PEG-Ab may provide a promising tool for the high-throughput analysis and rapid screening of small molecules in complex samples.

This work was co-supported by the Strategic Priority Research Program of the Chinese Academy of Sciences (XDB14010400), the National Natural Science Foundation of China (21107120, 21377141, 21422509, and 21107036) and the Excellent Young Scientist Grant of the Chinese Academy of Sciences.

\section{Notes and references}

1 (a) F. Simpkins, J. A. Czechowicz, L. Liotta and E. C. Kohn, Pharmacogenomics, 2005, 6, 647-653; (b) N. Tang, P. Tornatore and S. R. Weinberger, Mass Spectrom. Rev., 2004, 23, 34-44; (c) C. B. Liu, J. Biomed. Biotechnol., 2011, 2011, 245821.

2 (a) S. J. Guo and S. J. Dong, Chem. Soc. Rev., 2011, 40, 2644-2672; (b) K. S. Novoselov, V. I. Fal'ko, L. Colombo, P. R. Gellert, M. G. Schwab and K. Kim, Nature, 2012, 490, 192-200.

3 L. A. L. Tang, J. Z. Wang and K. P. Loh, J. Am. Chem. Soc., 2010, 132, 10976-10977.

4 (a) Q. Liu, J. B. Shi, J. T. Sun, T. Wang, L. X. Zeng and G. B. Jiang, Angew. Chem., Int. Ed., 2011, 50, 5913-5917; (b) Q. Liu, J. B. Shi and G. B. Jiang, Trends Anal. Chem., 2012, 37, 1-11.

5 (a) X. L. Dong, J. S. Cheng, J. H. Li and Y. S. Wang, Anal. Chem., 2010, 82, 6208-6214; (b) Q. Liu, M. T. Cheng and G. B. Jiang, Chem. - Eur. J., 2013, 19, 5561-5565; (c) M. H. Lu, Y. Q. Lai, G. N. Chen and Z. W. Cai, Anal. Chem., 2011, 83, 3161-3169; (d) Q. H. Min, X. X. Zhang, X. Q. Chen, S. Y. Li and J. J. Zhu, Anal. Chem., 2014, 86, 9122-9130.
6 B. Gulbakan, E. Yasun, M. I. Shukoor, Z. Zhu, M. X. You, X. H. Tan, H. Sanchez, D. H. Powell, H. J. Dai and W. H. Tan, J. Am. Chem. Soc., 2010, 132, 17408-17410.

7 (a) S. Dutta and S. K. Pati, J. Mater. Chem., 2010, 20, 8207-8223; (b) D. V. Kosynkin, A. L. Higginbotham, A. Sinitskii, J. R. Lomeda, A. Dimiev, B. K. Price and J. M. Tour, Nature, 2009, 458, 872-876; (c) D. K. James and J. M. Tour, Acc. Chem. Res., 2013, 46, 2307-2318. 8 (a) C. S. Xiang, P. J. Cox, A. Kukovecz, B. Genorio, D. P. Hashim, Z. Yan, Z. W. Peng, C. C. Hwang, G. D. Ruan, E. L. G. Samuel, P. M. Sudeep, Z. Konya, R. Vajtai, P. M. Ajayan and J. M. Tour, ACS Nano, 2013, 7, 10380-10386; (b) A. L. Higginbotham, D. V. Kosynkin, A. Sinitskii, Z. Sun and J. M. Tour, ACS Nano, 2010, 4, 2059-2069.

9 (a) K. C. Mccullough and R. E. Spier, Monoclonal Antibodies in Biology and Biotechnology: Theoretical and Practical Aspects, Cambridge University Press, London, UK, 1990; (b) A. Y. Kolosova, J. V. Samsonova and A. M. Egorov, Food Agric. Immunol., 2000, 12, 115-125; (c) J. Z. Shen, Z. Zhang, Y. Yao, W. M. Shi, Y. B. Liu and S. X. Zhang, Anal. Chim. Acta, 2006, 575, 262-266.

10 J. A. Mann, T. Alava, H. G. Craighead and W. R. Dichtel, Angew. Chem., Int. Ed., 2013, 52, 3177-3180.

11 X. Z. Zhou, Y. Y. Wei, Q. Y. He, F. Boey, Q. C. Zhang and H. Zhang, Chem. Commun., 2010, 46, 6974-6976.

12 Q. Liu, Q. F. Zhou and G. B. Jiang, Trends Anal. Chem., 2014, 58, 10-22.

13 (a) M. De, S. S. Chou and V. P. Dravid, J. Am. Chem. Soc., 2011, 133, 17524-17527; (b) T. Alava, J. A. Mann, C. Theodore, J. J. Benitez, W. R. Dichtel, J. M. Parpia and H. G. Craighead, Anal. Chem., 2013, 85, 2754-2759.

14 (a) J. H. Jung, D. S. Cheon, F. Liu, K. B. Lee and T. S. Seo, Angew. Chem., Int. Ed., 2010, 49, 5708-5711; (b) H. J. Lin, J. R. Huo, A. H. Zhang, Y. F. Liu, Q. M. Wang, Y. Cai, W. T. Ying, W. J. Qin, Y. J. Zhang and X. H. Qian, Analyst, 2012, 137, 3620-3623.

15 (a) L. Wang, Y. Zhang, X. Guo, Z. Duan and S. Wang, J. Agric. Food Chem., 2010, 58, 3265-3270; (b) T. Śniegocki, A. Posyniak and J. Żmudzki, Bull. Vet. Inst. Pulawy, 2006, 50, 353-357.

16 (a) C. Ardsoongnearn, O. Boonbanlu, S. Kittijaruwattana and L. Suntornsuk, J. Chromatogr. B: Anal. Technol. Biomed. Life Sci., 2014, 945-946, 31-38; (b) L.-J. Zhou, G.-G. Ying, S. Liu, J.-L. Zhao, F. Chen, R.-Q. Zhang, F.-Q. Peng and Q.-Q. Zhang, J. Chromatogr. A, 2012, 1244, 123-138; (c) S. Bayen, X. Yi, E. Segovia, Z. Zhou and B. C. Kelly, J. Chromatogr. A, 2014, 1338, 38-43.

17 Z. F. Li, L. S. Zhao, N. Liang, H. H. Chen and X. H. Hou, Anal. Methods, 2014, 6, 9045-9052. 